\title{
MedienPädagogik
}

Zeitschrift für Theorie und Praxis der Medienbildung

Jahrbuch Medienpädagogik 3.

Zweitveröffentlichung aus: Jahrbuch Medienpädagogik 3. (2003) Opladen: Leske+ Budrich. Herausgegeben von Ben Bachmair, Peter Diepold und Claudia de Witt.

\section{Einleitung: Jahrbuch Medienpädagogik 3}

\author{
Ben Bachmair, Peter Diepold und Claudia de Witt
}

Nimmt die wissenschaftlich arbeitende Medienpädagogik ihre Aufgabe angemessen wahr, obwohl sie nicht auf die Funktion von Medien in so bedrückenden, aktuellen Ereignissen wie dem Erfurter Amoklauf eingeht, sich auch nicht auf eine Medienschelte einlässt, wenn, wie die PISA-Studie 2000 zeigt, der Lernerfolg von Schülern und Schülerinnen schwindet? Es gibt aktuelle Meinungs- und Erörterungsforen, an denen sich Autoren des vorliegenden Jahrbuchs Medienpädagogik 3 beteiligt haben. ${ }^{1}$ Den Wissenschaftlerinnen und Wissenschaftlern der Kommission Medienpädagogik der Deutschen Gesellschaft für Erziehungswissenschaft (DGfE) geht es in diesem wissenschaftlich universitären Diskussionszusammenhang aber nicht vorrangig darum, praktikable Erklärungsansätze und ratgebende Hilfestellungen zu liefern. Im Vordergrund steht vor allem, Medienpädagogik in den vielseitigen Perspektiven von Bildungs-, Erziehungs- und Sozialisationstheorien zu entwickeln, um u.a. die Neugestaltung von Lernarrangements und Lernmethoden zu bedenken und zu fördern. So enthält dieses Jahrbuch aktuelle Ansätze aus der erziehungswissenschaftlichen Medienforschung. Dabei handelt es sich zum Teil um Beiträge, die auf dem Symposium „Neue Medien und erziehungswissenschaftliche Innovationsprozesse“ und in der Arbeitsgruppe „Neue Medien in der Lehrerausbildung - Impuls für Innovationen?“ der Kommission Medienpädagogik im Rahmen des DGfE-Kongresses „Innovation durch Bildung“ in München 2002 gehalten wurden. Darüber hinaus liegen weitere Beiträge aus der medienpädagogischen Forschung vor, die sowohl die Grundlagen als auch die anwendungsbezogene Forschung im Auge haben.

Die hier versammelten medienpädagogischen Beiträge distanzieren sich deutlich von bewahrpädagogischen Ansprüchen, sprechen vielmehr den Rezipienten von Medien eine aktive, kritische Rolle bei der Aneignung von Medieninhalten und beim Umgang mit Medien zu. Selbstverständlich sind die „neuen“ Medien nicht neutral gegenüber Rezeptions- und Aneignungs-

1 An dieser aktuellen Diskussion hat sich auch die Gesellschaft für Medienpädagogik und Kommunikationskultur (GMK) beteiligt (vgl. http://www.medienpaed.de/gmk/). 
formen, die sich mit den „alten“ Medien entwickelt haben. Deshalb stellt der erste Teil dieses Jahrbuchs neue Ansätze qualitativer medienpädagogischer Forschung vor. Sie diskutieren diese Veränderung jedoch nicht bewahrpädagogisch, normativ, sondern wenden sich einer sozial- und kulturwissenschaftlich orientierten Theorie mit der Absicht zu, Veränderungen in den Medien und in deren Aneignung herauszuarbeiten und pädagogisch zu bewerten. So skizziert Theo Hug neue Fragen und Aufgabenbereiche der medienpädagogischen Forschung, die auf ideologische Vorstellungen und Diktate verzichten, dafür sozial- und kulturwissenschaftlich orientiert sind. Wie ein solcher Forschungsansatz aussehen kann, verdeutlicht er an der Frage des „Weltwissens globaler Mediengenerationen“. Auch Hans-Dieter Kübler plädiert als Konsequenz aus den Ergebnissen der PISA-Studie dafür, Medienkompetenz mehr als bisher empirisch zu erforschen. Damit die wissenschaftliche Medienpädagogik ihre sozialwissenschaftliche Wende tatsächlich vollzieht, diskutiert er die Forschungsdefizite und schlägt dann vor, neue Zielformulierungen für Medienkompetenz aus einer originär pädagogischen Perspektive zu gewinnen, indem Schüler/-innen wie Mediennutzer/-innen als Subjekte im Zentrum pädagogischer Theorie und pädagogischen Handelns stehen. Weder Medien als Bedrohungsszenarien noch ein technologischer Verbesserungsoptimismus sind in der Lage, diese Subjektorientierung zu kompensieren. Mit der Verbindung von Medienkompetenz und Literalität setzen sich auch Ben Bachmair und Judith Seipold auseinander: Ihr Ansatzpunkt ist die Intertextualität als Verbindung medialer Angebote, die sie am Medium Fernsehen deutlich machen. Sie versuchen, die mit der Intertextualität verbundene Chance der Reflexivität und Orientierung herauszuarbeiten. Fernsehsender setzen bei den Zuschauern, auch denen der Generation der Kinder, eine Textkompetenz voraus, die mit einer auch schulisch definierten Lesekompetenz einhergeht. Obwohl sich die vertrauten medialen Darstellungsweisen verändern, womit die Idee des auswählenden Zuschauer in der vertrauten Form an Relevanz verliert, zielt innovatives Design neuer Femsehformate auf alte und neue Formen der Literalität, die in die Programmauswahl enthalten sind. In einer expliziten semiotischen Perspektive setzt sich Manfred Behr mit der visuellen Argumentation durch „Schlüsselbilder“ auseinander. Proposition, Argumentation, Extension und Intension sind die Bedeutungsdimensionen, über die sich Schlüsselbilder interpretieren lassen. Behr bietet der Medienpädagogik eine semiotische Methode der Bildinterpretation, um z.B. in der Schule eine den heutigen Medien angemessene Bild-Literalität zu fördern. Dazu geht er auf Schlüsselbilder zum Terrorangriff auf das World Trade Centre ein, die er für eine mögliche Arbeit in der Oberstufe erschließt.

Im zweiten Teil geht es um neue Ergebnisse und Erkenntnisse im Bereich der Medienbildung und Medienethik. So kommt Christina Schachtner aufgrund der Evaluation ihres Modellprojekts „Neue Medien in pädagogischen und psychosozialen Handlungsfeldern" zu der Kernaussage, dass das Lernen junger Erwachsener mit und durch neue Medien ein komplexer Konstrukti- 
ons-, Dekonstruktions- und Rekonstruktionsprozess ist. Dementsprechend erklärt sie mediales Lernen als Prozess der (Re-, De-)Konstruktion. Die sogenannte Grounded Theory dient als Matrix des methodischen Vorgehens und der Konstruktivismus als Erklärungsfolie. Differenziert werden mediale Konstruktion, ichbezogene Konstruktion und Weltkonstruktion, die sich mit einem integrativen Bildungsbegriff im Sinne Wolfgang Klafkis legitimieren und den Gedanken der Gestaltung in das Lernen hineintragen. Sylvia Buchen und Ingeborg Philipper gehen von einer medienbezogenen GenderPerspektive aus und stellen die Frage, wie sich Lernen, Bildung und Sozialisation durch den Umgang mit den neuen Medien verändern. Ihnen geht es auch um eine geschlechtsspezifisch sensitive Auseinandersetzung mit neuen Medien in unterschiedlichen Schulformen. Claudia de Witt stellt eine bildungstheoretische Perspektive zum Einsatz neuer Medien vor. Sie wählt dabei die pädagogische Perspektive des Pragmatismus John Deweys mit dem Ziel, selbstgesteuertes Lernen mit neuen Medien als Medienbildung zu verstehen. Im theoretischen Kontext des Pragmatismus bestimmt sie Medienbildung als einen inhaltlich orientierten Methodenprozess. Online-Ethnographie ist der Ansatz, den Winfried Marotzki zur Erforschung des Kulturraums Internet vorstellt und zur Differenzierung virtueller Communities anwendet. Mit der expliziten Hinwendung zur Ethnographie erklärt Marotzki aktuelle Formen medienvermittelter Kommunikation mit Hilfe einer lebensweltlichen Forschungstradition der Sozialwissenschaft. Rudolf Kammerl untersucht das Verhältnis medienbezogener moralischer Orientierungen Jugendlicher und medienpädagogischer Ethik. Eine medienpädagogische Diskursethik hilft ihm, Formen internetbasierter Kommunikation Jugendlicher über Chats zu befragen, wobei die kommunikativen Strukturen medialer Kommunikation wichtig sind. Als ein zentrales Thema der Medienforschung stellen Dorothee M. Meister und Uwe Sander die insbesondere in den 90er Jahren empirisch ausgerichtete Werbekompetenz vor, die sie verwenden, um exemplarisch in zentralen Fragen der Medienkompetenz einzuführen. Mit ihrer qualitativen und quantitativen Studie möchten sie konkret herausfinden, ob Werbekompetenz mit zunehmendem Alter und Bildungsgrad zunimmt.

Ein weiteres Forschungsgebiet der aktuellen Medienpädagogik beschäftigt sich damit, inwieweit digitale Lehr- und Lernangebote online oder offline die Vermittlung medienpädagogischer Kompetenz wirkungsvoll unterstützen und zugleich die spezifischen Chancen der neuen Medien erfahrbar machen können. Dazu bedarf es innovativer mediendidaktischer Modelle und Konzeptionen. Im dritten Teil entwickelt Bardo Herzig unter Mitarbeit von Silke von Grafe ein allgemein-didaktisches Konzept, in dem Multimedia-Scripts als besonderes (medien-)didaktisches Instrument zur Unterstützung von Reflexionsprozessen dienen. Das in diesem Beitrag verwendete Modell „pädagogischen Handelns in Alltagssituationen“ geht von der Problemlage aus, erziehungswissenschaftliches Wissen sei bei Lehramtsstudierenden für die Entwicklung pädagogischer Professionalität nur in geringem Maße relevant. Eine Reflexion, die Wissen über unterrichtliche Situationen mit eigenen, 
subjektiven Vorstellungen von Unterricht in Beziehung setzt, bietet auch Chancen zur Professionalisierung.

Es gibt eine erhebliche gesellschaftliche Dynamik, nicht nur um die neuen Medien in den Bildungsbereich hineinzutragen und ihnen hier eine wesentliche Funktion zu geben, sondern auch um das Konzept von Bildung zu beeinflussen. Wichtig sind hier Vorstellungen von instruktionaler Effizienz oder von einer Beschleunigung des Lernens, die eher von Fragen absehen, wie sich bei den aktuellen ökonomischen und medialen Veränderungen das Verhältnis der Generation der Kinder und Jugendlichen zu sich, zu ihrer sozialen und kulturellen Umwelt verändert, z.B. indem sie sich mediale Darstellungsformen als eigene Erlebnisweisen aneignen. Auch dieses sind medienpädagogische Fragen, mit denen sich Lehrer und Lehrerinnen in der Schule genauso als wesentliche Bildungsfrage auseinandersetzen sollten, wie das für Studierende möglich sein sollte. Wie wäre ein medienpädagogisch relevantes Lehramtsstudium zu gestalten? Wie könnte medienpädagogische Kompetenz in der Lehrerausbildung vermittelt werden? Was erwarten die Studierenden von der Lehrerausbildung? Sigrid Blömeke präsentiert zu diesem Fragenkomplex Ergebnisse einer medienpädagogisch akzentuierten empirischen Leistungsmessung und zieht Folgerungen für Lehre und Forschung in der Lehrerausbildung. Medienbezogene Kernaufgaben werden mit den Lernvoraussetzungen der Studierenden ins Verhältnis gesetzt. Gerhard Tulodziecki beschreibt die Gestaltung einer netzbasierten Lernumgebung für einen Fernstudiengang zu „Medien und Informationstechnologien in Erziehung, Unterricht und Bildung“. Grundlage bilden Entwicklungen zur Lehrerausbildung im Bereich neuer Medien, wie sie im Rahmen der Initiative „Bildungswege in der Informationsgesellschaft (BIG)“ entstanden sind. Charakteristisch ist die fall- bzw. handlungsorientierte Strukturierung der einzelnen Kursabschnitte. Einen Einblick in die didaktischen Entwicklungs- und Konzeptionsarbeiten eines teil-virtuellen Informatik-Praktikums (VIP) gibt Silvia Matalik. Sie berichtet aus einem laufenden Forschungsprojekt, das u.a. das Ziel hat, die Lehrqualität bei gleichzeitiger, längerfristig angelegter Ressourcenreduzierung zu erhöhen. Dabei bietet sich ein experimentelles und exploratives Lernen mit computerunterstützten Simulationsmedien an, deren didaktisches Design u.a. auf einer Lern- und Simulationsumgebung, einer virtuellen Betreuung und Videokonferenz aufbaut. 